

\section{RESUMEN}

Pau Casals fue el primer violonchelista en grabar la integral de las Suites de Bach, siendo este uno de sus legados musicales más relevantes. Esta grabación concluida en 1939 y la realizada por Anne Gastinel en 2007, presentan una interesante similitud: la duración de la Sarabande de la Suite N5, BWV 1011 , con una diferencia entre ambas inferior a los 3 segundos. A tenor de este escaso margen temporal, el objetivo del presente artículo es realizar mediciones de las duraciones a diferentes niveles o lupas en la Sarabande: las duraciones de cada grupo de 4 compases, las de cada compás y las de cada pulso, para posteriormente interpretar estos datos. Estas mediciones de la micro-agógica han sido efectuadas con el programa de análisis de audio Sonic Visualiser, y los datos obtenidos han sido analizados y comentados tanto por separado como en la comparación de ambos violonchelistas. Con todo ello, se muestran dos posibilidades distintas de ejecución de la agógica en unas grabaciones que, aunque en macro-agógica duran un tiempo similar, presentan sutiles convergencias y divergencias a medida que se agranda la lupa, dando cuenta del margen del intérprete en la ejecución.

\section{PALABRAS CLAVE}

Pau Casals, Anne Gastinel, Sarabande BWV 1011, Agógica, Sonic Visualiser.

\section{ABSTRACT}

Pau Casals was the first violoncellist in recording the whole corpus of Bach's Suites, which is indeed one of his most relevant musical legacy. Although several generations of musicians separtes this recording complited in 1939 from the one made by Anne Gastinel, there is one interesting similarity: the lenght of the recording of the Sarabande from the Fifth Suite, BWV 1011, whose time span does not exceed 3 seconds. Consequently, this article aims at measuring the Sarabande from three different levels: the lenght of each group of 4 bars, of each bar and of each beat. The measurement of the micro-agogic has been carried out using the audio-analysis program Sonic Visualiser. The data obtained have been interpreted both separately and comparing the performance of both cellists. If the recordings are examined closely, the end result uncover two different possibilities of performing the agogic and some convergences and divergences, even when at first sight it seems to be no difference. Finally, what becomes clear is the freedom of the player at the time of performing.

\section{KEYWORDS}




\title{
TRES LUPAS PARA EXAMINAR A PAU CASALS Y ANNE GASTINEL: ANÁLISIS DE LA AGÓGICA EN DOS INTERPRETACIONES DE LA SARABANDE BWV 1011 DE J.S. BACH.
}

\author{
Igor Saenz Abarzuza
}

Universidad Pública de Navarra

\section{CASALS Y GASTINEL}

\begin{abstract}
Como aquel amable conservador del hospital de San Juan, Casals ofrecía a su alumno una lupa - una lente que no agrandaba el espacio sino el tiempo. Era lo más adecuado para examinar un fragmento de una Suite de Bach: un solo compás, o medio movimiento, tocado con lentitud para poner bajo atento escrutinio la estructura de la frase, el cómo y por qué de cada inflexión dinámica, la cualidad precisa de la articulación, fácilmente pasada por alto a un tiempo más rápido. Con frecuencia se insta a los estudiantes de interpretación a "practicar despacio", principalmente para analizar las dificultades técnicas. El propósito de Casals era infinitamente más amplio; al aumentar la visión de un aspecto de la composición no solo lograba la atención máxima sobre todos los requisitos interpretativos, sino que sacaba a la luz las profundas raíces de la expresión desde la que se formula toda música (Blum 2000a, pp.111-112).
\end{abstract}

Considerado el violonchelista más importante de su generación, Pau Casals (El Vendrell, 1876 - San Juan, 1971) tuvo una influencia transcendental en el desarrollo del instrumento en el siglo XX, siendo este su legado más importante (Baldock 1994, p.52). Pero no se puede obviar que se trata de un artista de su generación, muy comprometido especialmente con la lucha por la paz a nivel global (dados los acontecimientos sociales de su tiempo), además de erigirse como un icono del catalanismo. Musicalmente, transcendió el estilo de su propia época para crear una nueva manera de tocar y de entender tanto el violonchelo como la música académica, ejerciendo también de director y compositor. Estudiar a Casals músico desde una óptica atemporal desdibujaría su legado, porque "(...) no se pueden valorar cabalmente los rasgos que definieron su estilo sin penetrar en las circunstancias que determinaron la estética de la interpretación en la segunda mitad del siglo XIX" (Zurita 2015, p.306).

Casals tuvo una educación musical poco habitual y hasta cierto punto autodidacta, marcada principalmente más por su entorno que por la tutela directa de un maestro de referencia en el violonchelo (Saenz, 2017a). Ya como artista consagrado, la industria discográfica que emerge con fuerza a inicios del Siglo XX le impulsó a realizar una grabación ciertamente atípica y revolucionaria en el mercado, la integral de las Suites para violonchelo Solo de Johann Sebastian Bach. Este acierto desde el punto de vista musical y comercial se produjo fruto de la insistencia del director de la EMI Fred Gaisberg, quien sedujo a Casals a pesar de las reticencias del violonchelista. De hecho, Fue el propio Gaisberg quien se encargó de la dirección artística tras la fusión en el año 1931 de HMV y Columbia Gramophone Europa. Casals y Gaisberg ya se conocían de proyectos anteriores, ya que fue el encargado de registrar los discos del trío Cortot-Thibaud-Casals en el periodo de 1926 a 1928. También logró convencer a Casals de grabar a la orquesta que creó en Barcelona en el año 1932.

A tenor de los testimonios, persuadir al violonchelista catalán para que registrara las Suites no fue en absoluto una tarea fácil: "si bien fue él quien convenció a Casals para que volvie- 
ra a un estudio de grabación, no está claro cómo lo consiguió" (Baldock, 1994, p.202). Es conocida la aversión que Casals tenía al hecho de grabar, llegando a menudo a no reconocerse a sí mismo en sus propias grabaciones (Corredor. 1975, p.251). Un verdadero odio reconocido por él hacia los voluminosos aparatos de grabación de aquella época, el "monstruo de acero" (Siblin, 2011, p.111) y una manifiesta disconformidad con el sonido de los discos, por su diferencia con el directo (Eisenberg, 1943, p.2). A todo esto, se suma la presión a la que el mismo Pau Casals se sometía: "(...) el hecho de que en los discos todo quede grabado impone al artista una verdadera servidumbre $y$, lo que es peor, una servidumbre peligrosa para su inspiración y su sentimiento musical. Actualmente, la mecanización nos acecha por todas partes" (Corredor, 1975, p.226).

Sin pretenderlo, esta grabación marcó un antes y un después en su carrera, en su devenir y en su propio legado musical. La figura de Casals ha quedado irremediablemente ligada a las Suites de Bach, atribuyéndole en un tono ciertamente legendario su re-descubrimiento gracias a aquel hallazgo feliz de la partitura en una tienda de música de Barcelona (Saenz, $2017 b)$. Como ejemplo del proceso de preparación al que se sometió, lo que cuenta Joan Alavedra (1975, p.323):

Para las Suites de Bach se prepara con mucha intensidad. Casals me había hablado del sudor que a veces cae sobre el violonchelo mientras el artista toca, o el de los dedos, que resbala sobre las cuerdas. Esta vez, cuando ha de impresionar las Suites, tiene los dedos de la mano izquierda en Sangre. "Esto pasa, no es nada." Está nervioso, pero sonríe.

De la histórica grabación de Casals efectuada entre los años 1936 a $1939^{1}$ a la realizada para el sello Naïve por la concertista y pedagoga francesa Anne Gastinel² (Tassin-la-Demi-Lune, 1971), hay varias generaciones de violonchelistas: hoy en día, se puede acceder a decenas de grabaciones de diversas escuelas interpretativas, muchas de ellas antagónicas. Gastinel y Casals nunca llegaron a conocerse, ya que cuando Casals murió en 1973, Gastinel apenas tenía 2 años. La violonchelista francesa acometió la tarea en 2007, por lo que a ambas grabaciones las separa un margen de 68 años. Con 37 años, Gastinel registró la integral de las Suites mucho antes de lo que lo suelen hacer otros músicos, lo que apunta, según la revisión realizada para Gramophone por Lindsay Kemp, a que en un par de décadas pudiera realizar otra ya con una visión diferente. Ante un Bach que pudo estar en la treintena cuando escribió las Suites, y en respuesta a Kemp, Charlotte Gardner dice en su artículo de The Guardian "żdemasiado joven para tocar Bach? No hay límite de edad para tocar las Suites" 3: "Żquizás nos estamos tomando estas obras más en serio que el propio Bach? La suposición general es que se necesitan años de sabiduría para interpretar las Suites satisfactoriamente" ${ }^{\prime 4}$.

Al margen de esta cuestión, el punto común de ambos violonchelistas está en Francia: por una parte, Casals pasó años en este país, concretamente en Prades (si bien antes había residido en París). Gastinel, por su parte, además de ser francesa de nacimiento, se formó musicalmente en el país galo donde actualmente enseña y vive, concretamente en los Conservatorios $\mathrm{Na}$ cionales de París y Lyon (centro del que es profesora desde 2003). Con una dilatada carrera concertística, pedagógica y discográfica, a los dos intérpretes les une también que, en 1997. Gastinel fue elegida para tocar por un año el Matteo Goffriller de Casals, cedido por su esposa, Marta Casals-Istomin. Para la grabación de las Suites, Anne Gastinel usó un Testore de 1690.

\footnotetext{
1 Pablo Casals: tema (5 de abril de 2017). Cello Suite No. 5 in C Minor BWW 1011 : N. Sarabande [Archivo de vídeo]. Recuperado de hitlps://mmw.youtube.com/ watch? $=$ wO_Y8uqYXU

2 My Classical Playlists (23 de abril de 2014). Anne Gastinel - Suite n5 en Do Mineur BWW 1011 (Sarabande) [Archivo de vídeo]. Recuperado de hitips://unww.youtube. $\mathrm{com} /$ watch? $\mathrm{v}=51$ kjoi2tyaA

3 "Too young to play Bach? There is no need for an age limit on playing the cello suites".

4 "Are we, I'm wondering, taking these works more seriously than Bach did? The general assumption now is that one needs the wisdom of years to interpret them successfully".
} 


\section{LA SARABANDE BWV 1011: DURACIÓN Y ESTRUCTURA}

Llama poderosamente la atención la duración de las grabaciones que Casals y Gastinel realizaron de la Sarabande, ya que estas dos versiones duran prácticamente lo mismo: la grabación que Gastinel, "(...) de una amplitud fascinante" $\mathbf{5}$ según la revisión de Joanne Talbot para Strad, dura 2 minutos y 44,977 segundos, mientras que la de Casals, 2 minutos y 47,626 segundos. La diferencia es tan solo de 2,649 segundos. Esta particular igualdad no se puede encontrar en otras grabaciones comerciales, que como se puede observar, todas tienen una duración significativamente mayor. Por citar algunos ejemplos de violonchelistas de referencia, la grabación que YoYo Ma hizo en 2018, tiene una duración de $3: 10$, y 3:18 en la que hizo en 1983. De este mismo año es la que Paul Tortelier registró con una duración de 3:17. En el caso de Anner Bylsma, su Sarabande duró 3:17 en la primera grabación realizada con criterios historicistas, y 3:13 en la segunda grabación que hizo en 1992. En el caso de Mstislav Rostropovich, su Sarabande de 1995 dura 3:51, y la de Pieter Wispelwey de 2012, 3:41. La versión de Ralph
Kirshbaum de 1994 dura 4:20, y 3:37 la que Janos Starker registró en 1997. Mischa Maisky la grabó dos veces, una en 1985 con una duración de 4:54 y otra en 1999, con una Sarabande de 4:15. En el Caso de los franceses Bruno Cocset y Jean-Guihen Queyras, la del primero dura 3:55 y es de 2001, mientras que la del segundo, de 2007, dura 3:206 . Como puede observarse a tenor de estas duraciones, la de Gastinel es la que más se acerca en duración a la primera grabación referencial realizada por Casals.

La Sarabande7, como puede verse en la Imagen $N^{0} 1$, está dividida en dos partes que se repiten, $A$ y B. La parte A consta de 8 compases, y B de 12. Como tanto Casals como Gastinel realizan las dos repeticiones, la forma se establece como A-A'/B-B'. Son en total 108 notas, 100 de ellas corcheas, 7 negras y una blanca con puntillo en el final de $A$.

\section{Imagen 1}

Sarabande de la Suite N5, transcripción basada en las copias de Anna Magdalena Bach y Johann Peter Kellner.

\section{Sarabande}
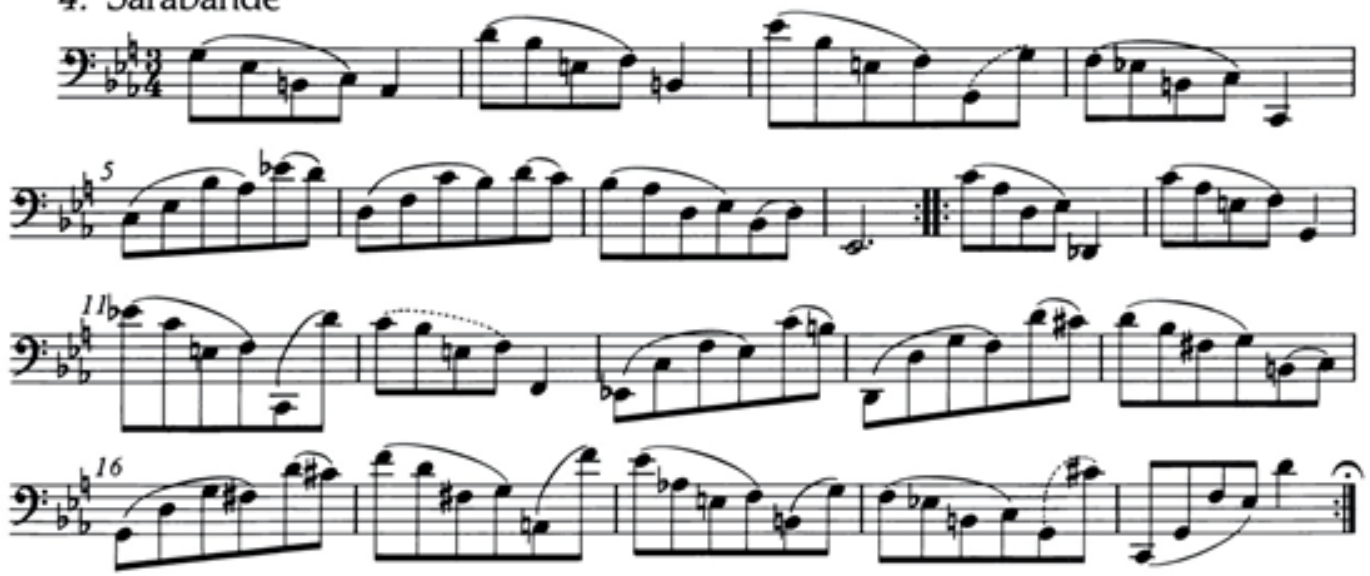

\footnotetext{
5 "(...) while the Sarabande of the same suite has a mesmerising spaciousness".

6 En la siguiente playlist de Spotify se pueden escuchar todas las grabaciones referidas: hitps://open.spotify.com/user/chelowsky/playlist/3yHcHYYOLWYYA78kqnnxbKi? si=9VqODCiaSZ6FFILwte9pzQ

7 La Suite No5 está escrita para ser tocada con Scordatura, quedando la afinación del violonchelo de la siguiente manera: Sol-Re-Sol-Do. En las transcripciones de la parlitura original perdida realizadas por Anna Magdalena Bach y Johann Peter Kellner, la Suite NN5 se presenta escrita para ser tocada como si la scordatura no se hubiese producido: es decir, como si el Sol de la primera cuerda siguiese siendo un La. Este hecho da pistas sobre en qué cuerdas y posiciones pensó Bach que debía ser interpretada la obra, a la vez que facilita su lectura para los violonchelistas acostumbrados a la afinación estandarizada del instrumento: La-Re-Sol-Do.
} 


\section{OBJETIVO Y METODOLOGÍA}

El objetivo principal de la investigación que se presenta es comparar la interpretación de Casals con la de Gastinel en la Sarabande de la Suite N5 BWV 1011 para violonchelo solo de Johann Sebastian Bach. Concretamente, se pone el foco en la agógica, diferenciando la macro-agógica de la micro-agógica. Para la obtención de los datos de las duraciones de ambas interpretaciones, se ha usado el programa de análisis de software libre Sonic Visualiser en su versión 3.2.1. Se presenta un análisis computacional y sus resultados, así como una interpretación de los mismos. Los datos se muestran en tres lupas o niveles de análisis micro-agógicos: los grupos de $4 \mathrm{com}$ pases, el compás, y el pulso. Adicionalmente, se analizan las duraciones macro-agógicas referidas al total de la obra y a las partes $A-A^{\prime}$ y $B-B^{\prime 8}$.

Para realizar este análisis de la manera más exhaustiva posible, se ha procedido a medir la duración de cada una de las 108 notas de la Sarabande y sus repeticiones en las grabaciones de Pau Casals y Anne Gastinel. Para ello, se han colocado marcas en el inicio y final de cada nota, bajando la velocidad de reproducción hasta un $12,5 \%{ }^{\circ}$ con el objetivo de ser lo más preciso posible. Posteriormente, mediante tablas de hojas de datos, se ha procedido a obtener el resto de los datos que se presentan en las siguientes líneas, a partir del primer dato de la duración de cada nota extraído directamente de Sonic Visualiser ${ }^{\mathbf{1 0}}$.

\section{RESULTADOS DEL ANÁLISIS COMPARATIVO}

\section{Interpretación de datos macro-agógicos}

Antes de abordar la interpretación de la música, un breve comentario acerca de los silencios iniciales y finales de las dos grabaciones: se entiende que estos lapsos de tiempo cuentan con el beneplácito del intérprete. Dicho esto, en la grabación de Gastinel, el silencio inicial dura 0,743 y el final 3,413 , mientras que en la de Casals, el inicial dura 0,775 y el final, 3,489 . Resulta destacable esta igualdad entre ambas grabaciones. El silencio inicial cuenta con una escasa diferencia que, poniendo el corte en el decisegundo, sería de 0,0: al milisegundo, ese lapso es de 0,032 '1. Acerca del silencio final, la interpretación del resultado es similar con una diferencia escasa de 0,076. Este silencio final resulta más llamativo, ya que esta duración casi quintuplica a la del inicio. Incluso así, al decisegundo la diferencia es de 0,0 .

En cuanto a la duración total de la $A-A^{\prime}$ y de $B-B^{\prime}$, en la grabación de Pau Casals, A dura 34,031 y $A^{\prime}, 32,166$. La parte B dura 48, 107, y $B^{\prime}, 48,939$. En la grabación de Anne Gastinel, $A$ dura 31,333 y $A^{\prime}, 31,8$. La parte B dura 46,785 y $B^{\prime}, 51,015$. Por tanto, mientras que en la grabación de Anne Gastinel $A^{\prime}$ y $B^{\prime}$ duran más que $A$ y $B$, en la de Pau Casals $A$ dura más que $A^{\prime}$, y $B^{\prime}$ dura más que $B$. Se puede observar cómo en Casals la diferencia entre $A$ y $A^{\prime}$ es de 1,865 segundos a favor de $A$, y entre $B$ y $B^{\prime}$ la diferencia es de 0,832 segundos a favor de $B$, casi un segundo de diferencia entre ambas. En la grabación de Anne Gastinel, la diferencia entre $A$ y $A^{\prime}$ es de 0,467 en favor de $A^{\prime}$, mientras que la diferencia entre $B$ y $B^{\prime}$ es de 4,23, en esta ocasión en favor de B. Se muestra la gran diferencia de duración entre $B$ y $\mathrm{B}^{\prime}$ en la interpretación de Anne Gastinel, cuando en la de Pau Casals no llega al segundo.

Comparando ambas versiones, se puede ver que en cuanto a la parte $A$, la grabación de Casals dura 2,698 más que la de Gastinel, que es la mayor diferencia entre ambas interpretaciones. La parte $A^{\prime}$, con una desigualdad de 0,366, marca la menor diferencia. La parte $B$ de Casals vuelve a durar más, con 1,322, mientras que la parte $B^{\prime}$, se muestra a favor de la versión de Anne Gastinel de 2,076, la

\footnotetext{
8 En este arí́ílo no se analizan las duraciones nota por nota. Para un análisis exdusivo de la versión que Casals registró de la Sarabande, en Saenz (2018a) se expuso cómo Casals alargaba mayoritaniamente la duración de las notas del inicio o fin del compás, con una clara relación entre el análisis ammónico-estructural yel interpretativo. Se conduyó que las diferentes combinaciones que se crean aportan variedad a la vez que respetan la partitura de la Sarabande de Bach.

- Se trata de la mínima velocidad que permite Sonic Visualiser, que tiene un margen que va desde 12,5\% hasta 800\%. Este cambio en la velocidad no afecta a los otros parámetros del sonido registrado en la señal de audio como son el timbre, la altura y la intensidad.

10 Para conocer con profundidad la metodología seguida con Sonic Visualiser tanto en esta investigación como en previas, consultar Saenz (2018b).

11 En todo el proceso de análisis, se han utilizado mediciones acotadas al milisegundo por ser las que ofrece Sonic Visualiser.
} 
única que dura más en la interpretación de la violonchelista. Es estos datos macro-agógicos, se pueden ver claramente dos propuestas de interpretación muy diferenciadas.

\section{PRIMERA LUPA:}

\section{LOS GRUPOS DE 4 COMPASES}

Las dos partes de la Sarabande se pueden subdividir en un total de 5 grupos de 4 compases, 2 en la parte $A-A^{\prime}$ y 3 en B-B'. La primera lupa micro-agógica se establece en las diferencias y similitudes entre los dos intérpretes por separado y en comparación de ambos, en el tiempo que dedican a la interpretación de cada uno de estos grupos.
En la siguiente Tabla $\mathrm{N}^{0} 1$ se puede observar la duración de estos grupos ajustada al milisegundo en la interpretación de Casals, diferenciando con el nombre "Duración primera int." y "Duración repetición" las dos veces que se ejecutan la Parte A y la Parte B de la obra tanto en la versión de Casals como en la de Gastinel.

\section{Tabla 1}

Duraciones de los grupos de 4 compases en Casals.

\begin{tabular}{cccc} 
Compases & Duración primera int. & Duración repetición & Diferencia \\
\hline cc. $1-4$ & 17,833 & 16,8 & 1,033 \\
\hline cc. $5-8$ & 16,198 & 15,366 & 0,832 \\
\hline cc. $9-12$ & 16,172 & 15,568 & 0,604 \\
\hline cc. $13-16$ & 15,527 & 15,796 & $-0,269$ \\
\hline cc. $17-20$ & 16,408 & 17,575 & $-1,167$ \\
\hline
\end{tabular}

Como se puede ver en la cuarta columna, en la interpretación de Casals de la Parte A hay una prevalencia de duración mayor en la primera interpretación que en la repetición de A. En B, la tendencia se invierte, con especial énfasis en el último grupo de 4 compases debido al ritardando final que se realiza en la repetición de $B$ para concluir la pieza. Es en este último grupo donde se puede ver una mayor diferencia. Como grupo de duración larga, destaca el primero de A con una duración de 17,833, y el más breve, el segundo de $A^{\prime}$ con 15,366.

En la siguiente tabla, se puede ver la misma información que en la tabla anterior, pero esta vez de la interpretación de Anne Gastinel:

\section{Tabla 2}

Duraciones de los grupos de 4 compases en Gastinel.

\begin{tabular}{cccc} 
Compases & Duración primera int. & Duración repetición & Diferencia \\
\hline cc. $1-4$ & 16,179 & 16,324 & $-0,145$ \\
\hline cc. $5-8$ & 15,051 & 15,362 & $-0,311$ \\
\hline cc. $9-12$ & 15,16 & 16,199 & $-1,039$ \\
\hline cc. $13-16$ & 15,446 & 15,69 & $-0,244$ \\
\hline cc. $17-20$ & 16,179 & 19,126 & $-2,947$ \\
\hline
\end{tabular}


Como puede observarse en la Tabla $\mathrm{N}^{\circ} 2$, en mayor o menor medida, la repetición tanto de A como de B tienen en todos los casos una duración mayor que la primera interpretación. En el caso del último grupo, es muy notable la prevalencia de la repetición. También se puede ver cómo en el final de A, la segunda interpretación tiene una notable mayor duración por encima del segundo.

A diferencia de Casals, en la interpretación de Gastinel no es el primer grupo el que destaca como largo, sino el último de $B^{\prime}$ y por tanto el que cierra la interpretación de la Sarabande. En comparación a la interpretación de Casals, se puede ver que en la de Gastinel, los extremos se acentúan: tanto el grupo más largo, el último de $\mathrm{B}^{\prime}$, como el más corto, los compases 5 a 8 de la primera interpretación con una duración de 15,051, son ambos de duraciones más largas en el primer caso y breves en el segundo. Así, Gastinel realiza mayores diferencias en los sub-grupos de 4 compases. El cuarto grupo de 4 compases (compases 13 a 16) presenta tanto en la primera interpretación como en la repetición escaso margen de diferencia: 8 décimas en la primera y 10 en la repetición. Muy cercana es la similitud entre la repetición de los compases 13 a 16 en ambos intérpretes, con una diferencia ajustada de 10 décimas. Así pues, este grupo es que mayores similitudes presenta entre la versión de Casals y la de Gastinel. Quizás la coincidencia más destacable sea la de la repetición del segundo grupo de 4 compases, los compases 5 a 8 , donde ambas interpretaciones se llevan un margen de 4 milisegundos. Como se puede ver, más que buscar similitudes, nos encontramos ante dos modelos diferentes, aunque no opuestos, por marcar duraciones no comparables y con tendencias diferentes en sus números pero sobre todo, en las tendencias. La de Casals, si destaca por algo, es por una mayor variedad, siendo los grupos más largos el de los primeros 4 compases de la primera interpretación de $A$, y el final de la obra en la repetición de B. En cambio, Gastinel se toma más tiempo en los finales de las partes $A$ y $B$ de la repetición. Como se ha mencionado, en esta primera lupa y dentro de la variedad que presentan ambas interpretaciones, se puede ver mayor igualdad en la de Casals y un mayor grado de variedad en las duraciones de la versión de Gastinel.
Para acabar con esta primera lupa, en la Tabla $\mathrm{N}^{\circ} 3$ se puede ver la diferencia de los grupos restando la duración de cada grupo en la interpretación de Gastinel a la de Casals. Por tanto, un resultado positivo implica mayor duración en la de Casals, y un resultado en negativo, una mayor duración en la interpretación de Gastinel.

\section{Tabla 3}

Duraciones de los grupos de 4 compases en Gastinel.

\begin{tabular}{ccc} 
Compases & $\begin{array}{c}\text { Duración } \\
\text { primera int. }\end{array}$ & $\begin{array}{c}\text { Duración } \\
\text { repetición }\end{array}$ \\
\hline cc. 1-4 & 1,654 & 0,476 \\
\hline cc. 5-8 & 1,147 & 0,004 \\
\hline cc. 9-12 & 1,012 & $-0,631$ \\
\hline cc. 13-16 & 0,081 & 0,106 \\
\hline cc. 17-20 & 0,229 & $-1,551$ \\
\hline
\end{tabular}

En búsqueda de convergencias, el grupo de los compases 13 a 16 de la primera interpretación y el grupo de los compases 5 a 8 de la repetición tienen una diferencia que, acotada al decisegundo, sería de 0,0. Todavía más: en el caso de la repetición de los compases 5 a 8 , si la acotación fuera al centisegundo, sería de 0,00 . Se puede concluir que, virtualmente, ambos grupos tienen una misma duración, independientemente de lo que pase dentro del grupo o en el sumatorio con el resto de la parte.

\section{SEGUNDA LUPA: LOS COMPASES.}

A continuación, en la Tabla $\mathrm{N}^{\circ} 4$, se presentan los datos referidos a la duración de cada uno de los compases en la versión de Casals, diferenciando en dos columnas separadas la primera interpretación de la repetición. La última columna, al igual que en las tablas anteriores, está reservada a la diferencia de las columnas 2 y 3. 
Tabla 4

Duraciones de los compases en Casals.

\begin{tabular}{|c|c|c|c|}
\hline Compases & Duración primera int. & Duración repetición & Diferencia \\
\hline c. 1 & 5,068 & 4,511 & 0,557 \\
\hline c. 2 & 4,618 & 4,296 & 0,322 \\
\hline c.3 & 3,797 & 3,758 & 0,039 \\
\hline c. 4 & 4,35 & 4,235 & 0,115 \\
\hline c. 5 & 3,979 & 3,737 & 0,242 \\
\hline c. 6 & 3,805 & 3,552 & 0,253 \\
\hline c.7 & 4,003 & 3,852 & 0,151 \\
\hline c.8 & 4,411 & 4,225 & 0,186 \\
\hline c.9 & 4,398 & 4,02 & 0,378 \\
\hline c. 10 & 3,915 & 3,813 & 0,102 \\
\hline c. 11 & 3,799 & 3,852 & $-0,053$ \\
\hline c. 12 & 4,06 & 3,883 & 0,177 \\
\hline c. 13 & 4,092 & 4,382 & $-0,29$ \\
\hline c. 14 & 3,599 & 3,663 & $-0,064$ \\
\hline c. 15 & 3,756 & 3,775 & $-0,019$ \\
\hline c. 16 & 4,08 & 3,976 & 0,104 \\
\hline c. 17 & 4,113 & 4,265 & $-0,152$ \\
\hline c. 18 & 3,93 & 3,948 & $-0,018$ \\
\hline c. 19 & 3,856 & 4,222 & $-0,366$ \\
\hline c. 20 & 4,509 & 5,14 & $-0,631$ \\
\hline
\end{tabular}

En la interpretación de Pau Casals, el compás 11 tiene una ligera mayor duración en $\mathrm{B}^{\prime}$, tan ligera que incluso en micro-agógica, la diferencia no es destacable: 0,0529 a favor de $B^{\prime}$. Algo parecido sucede con los siguientes tres compases: en el compás 14, en $B^{\prime}$ dura 0,064 más que en $B$. En el compás 15, en $B^{\prime}$ dura 0,019 más que en $B$, y finalmente en el compás 18, hay una diferencia de 0,017 a favor de $B^{\prime}$ respecto a $B$. Las diferencias más significativas entre la primera interpretación y la repetición se pueden observar en los compases 1,2 y 9 en favor de la primera interpretación y en el compás final de la repetición, el 20. Por su escaso margen, en el compás 3, la imperceptible diferencia juega a favor de $A$ frente a $A^{\prime}$ por 0,039 segundos. Por tanto, de los 20 compases, en la primera parte todos los compases duran más en $A$ que en $A^{\prime}$, con el matiz mencionado del compás 3. En la segunda parte, cuatro compases tienen mayor duración en $B$ y ocho la tienen en $B^{\prime}$, con los comentarios señalados. Así, en la interpretación que Casals realiza de la parte $A$, todos los compases duran más en la primera interpretación que en la repetición. Respecto a la parte $B$, de los 12 compases, en cuatro se mantiene esta tendencia expresada en $A$, pero en ocho la tendencia se invierte.

Mirando el mismo parámetro en la interpretación de Gastinel presentada en la Tabla N5, no se observa una tendencia tan clara. Aquí no existe un patrón como tal: en la parte $A$ son 
cuatro los compases que duran más en la primera interpretación y cuatro en la repetición. En la parte B, son tres los que tienen una duración mayor en en la primera interpretación aunque dos de ellos con un escaso margen que acotado al decisegundo sería de 0,0. En esta versión de Anne Gastinel, el compás 2 tiene una imperceptible diferencia a favor de $A$ frente a $A^{\prime}$ de 0,014 , caso similar al del com- pás 4 con 0,072 esta vez a favor de $A^{\prime}$ frente a A. En el compás 15 la diferencia es de 0,07 a favor de B, y en el compás 16, 0,041 a favor también de B. Mirando el parámetro del compás en esta segunda lupa, Gastinel realiza mayores duraciones en la repetición de $A$ y $B$ que en la primera interpretación.

Tabla 5

Duraciones de los compases en Gastinel.

\begin{tabular}{|c|c|c|c|}
\hline Compases & Duración primera int. & Duración repetición & Diferencia \\
\hline c. 1 & 4,278 & 4,152 & 0,126 \\
\hline c. 2 & 3,979 & 3,965 & 0,014 \\
\hline c. 3 & 3,464 & 3,677 & $-0,213$ \\
\hline c. 4 & 4,458 & 4,53 & $-0,072$ \\
\hline c. 5 & 3,615 & 4,062 & $-0,447$ \\
\hline c. 6 & 3,502 & 3,281 & 0,221 \\
\hline c.7 & 3,636 & 3,908 & $-0,272$ \\
\hline c. 8 & 4,298 & 4,111 & 0,187 \\
\hline c. 9 & 4,087 & 4,259 & $-0,172$ \\
\hline c. 10 & 3,521 & 4,097 & $-0,576$ \\
\hline c. 11 & 3,39 & 3,809 & $-0,419$ \\
\hline c. 12 & 4,162 & 4,034 & 0,128 \\
\hline c. 13 & 4,101 & 4,258 & $-0,157$ \\
\hline c. 14 & 3,687 & 3,885 & $-0,198$ \\
\hline c. 15 & 3,77 & 3,7 & 0,07 \\
\hline c. 16 & 3,888 & 3,847 & 0,041 \\
\hline c. 17 & 4,314 & 4,484 & $-0,17$ \\
\hline c. 18 & 3,705 & 3,829 & $-0,124$ \\
\hline c. 19 & 3,689 & 4,581 & $-0,892$ \\
\hline c. 20 & 4,471 & 6,232 & $-1,761$ \\
\hline
\end{tabular}

Tanto la versión de Pau Casals de la Sarabande como la de Anne Gastinel, coinciden en el compás de mayor duración, el último de la repetición que pone fin a la obra. No coinciden en el compás de menor duración, que es el 11 con 3,39 en la primera interpretación de
Gastinel, y el compás 6, con una duración de 3,552 en la repetición de la versión de Casals.

Mirando diferencias destacables por encima del medio segundo, Casals toca el primer compás 0,557 más largo en A, y el compás 20 
dura más en $\mathrm{B}^{\prime}$ con una diferencia de 0,631. Por tanto, las mayores diferencias ya destacables se dan tan solo en el primer y último compás. En la versión de Gastinel no se repite esta idea interpretativa: aquellos compases que se acercan a medio segundo de duración son destacables, como la diferencia de 0,447 del compás 5 a favor de $A^{\prime}$, el compás 10, con 0,576 a favor de $B^{\prime}$ y el compás 11, a favor también de $B^{\prime}$. Se acerca al segundo de diferencia el compás 19, con 0,892 a favor de $\mathrm{B}^{\prime}$ y el 1'761 del compás 20 con una duración mayor también en $B^{\prime}$. Por tanto, en la segunda lupa y tal y como sucedía en la primera, se sigue observado una mayor polarización de los extremos en la versión de Gastinel con respecto a la de Casals.

Para acabar con la segunda lupa, en la Tabla $N^{0} 6$ se comparan las duraciones de los compases esta vez entre Casals y Gastinel, habiendo restado la duración de Casals a la de Gastinel. Así, un resultado positivo determina una mayor duración en la interpretación del violonchelista catalán y viceversa.

\section{Tabla 6}

Diferencias entre ambas interpretaciones respecto a la duración de los compases.

\begin{tabular}{ccc} 
Compases & $\begin{array}{c}\text { Duración } \\
\text { primera int. }\end{array}$ & $\begin{array}{c}\text { Duración } \\
\text { repetición }\end{array}$ \\
\hline c. 1 & 0,79 & 0,359 \\
\hline c. 2 & 0,639 & 0,331 \\
\hline c. 3 & 0,333 & 0,081 \\
\hline c. 4 & $-0,108$ & $-0,295$ \\
\hline c. 5 & 0,364 & $-0,325$ \\
\hline c. 6 & 0,303 & 0,271 \\
\hline c. 7 & 0,367 & $-0,056$ \\
\hline c. 8 & 0,113 & 0,114 \\
\hline c. 9 & 0,311 & $-0,239$ \\
\hline c. 10 & 0,394 & $-0,284$ \\
\hline c. 11 & 0,409 & 0,043 \\
\hline c. 12 & $-0,102$ & $-0,151$ \\
\hline c. 13 & $-0,009$ & 0,124 \\
\hline c. 14 & $-0,088$ & $-0,222$ \\
\hline c. 15 & $-0,014$ & 0,075 \\
\hline & &
\end{tabular}

\begin{tabular}{lll}
\hline c. 16 & 0,192 & 0,129 \\
\hline c. 17 & $-0,201$ & $-0,219$ \\
\hline c. 18 & 0,225 & 0,119 \\
\hline c. 19 & 0,167 & $-0,359$ \\
\hline c. 20 & 0,038 & $-1,092$ \\
\hline
\end{tabular}

Como se puede observar en los resultados, en 14 ocasiones de 20, Casals toca más largos los compases en la primera interpretación, mientras que en la segunda, son 10 y 10, mitad y mitad. En la primera interpretación, los compases 13, 14 y 20 tienen una duración que acotada al decisegundo es de 0,0 , virtualmente iguales 0 , en todo caso, con una imperceptible diferencia a tiempo real. Destaca, en la búsqueda de igualdades si las hubiere, el compás 13, con una duración de 0,003 en favor de la interpretación de Gastinel, también (o todavía más, si cabe) virtualmente igual a la de Casals. En la repetición, las igualdades de encuentran en los compases 3, 7, 11 y 15. En cuanto a la mayor divergencia, en el último compás de la repetición la diferencia micro-agógica es abismal, por encima del segundo en la versión de Gastinel. En datos acotados al milisegundo, una diferencia de esta envergadura es más que notable.

\section{TERCERA LUPA: LOS PULSOS}

La tercera y última lupa se establece en los pulsos, 3 por cada compás. A excepción del compás 8 donde hay una blanca con puntillo, el resto de compases se pueden hacer mediciones diferenciadas por pulsos. Con el fin de obtener el dato de ese compás 8 también, se ha subdividido la blanca con puntillo en tres partes iguales. Para la correcta localización de los datos, se ha colocado una primera columna con el número de compás, y una segunda con el número de pulso (1, 2 o 3).

Respecto a interpretación de Pau Casals y como se puede observar en la Tabla $\mathrm{N}^{0} 7$, la mayor diferencia se puede ver en la primera nota, muy notable, de 0,412 entre la primera interpretación y la repetición. Como dato llamativo pero casi anecdótico, el tercer pulso del tercer compás tiene la misma duración ajustada al milisegundo en ambas. 
De los 24 pulsos, tan solo en 5 la duración es mayor en a la repetición. Y lo que es más destacable: estas duraciones en favor de B marcan 0,0 si se limitara al decisegundo. En 2 ocasiones, con una marca 0,00 si se limitara al centisegundo, se aprecian diferencias tan solo al milisegundo. Así, puede determinarse que virtualmente supone una no intencionalidad en alargar visiblemente la interpretación en la repetición. De todos modos, esta mayor duración en la primera interpretación presenta valores de 0,0 al decisegundo en 15 ocasiones. Con este dato, en 15 casos (incluyendo el tercer pulso del compás 4) no parece que Casals tenga una intencionalidad en diferenciar ambas interpretaciones de forma global en lo que respecta a los pulsos. En los casos donde sí se aprecian diferencias, destaca la primera nota con una mayor duración de 0,415 y el tercer pulso del compás 7 con 0,201. Este pulso es previo a la nota final de la parte $A$, y Casals opta por alargarlo decididamente antes de la repetición y no tras ella.

Tabla 7

Duraciones de los pulsos en Casals. Parte A.

\begin{tabular}{|c|c|c|c|c|}
\hline Compases & Pulso & Duración primera int. & Duración repetición & Diferencia \\
\hline c. 1 & 1 & 1,932 & 1,52 & 0,412 \\
\hline c. 1 & 2 & 1,328 & 1,334 & $-0,006$ \\
\hline c. 1 & 3 & 1,808 & 1,657 & 0,151 \\
\hline c. 2 & 1 & 1,5 & 1,43 & 0,07 \\
\hline c. 2 & 2 & 1,357 & 1,256 & 0,101 \\
\hline c. 2 & 3 & 1,761 & 1,61 & 0,151 \\
\hline c.3 & 1 & 1,297 & 1,244 & 0,053 \\
\hline c. 3 & 2 & 1,146 & 1,16 & $-0,014$ \\
\hline c. 3 & 3 & 1,354 & 1,354 & 0 \\
\hline c. 4 & 1 & 1,235 & 1,23 & 0,005 \\
\hline c. 4 & 2 & 1,293 & 1,244 & 0,049 \\
\hline c. 4 & 3 & 1,822 & 1,761 & 0,061 \\
\hline c. 5 & 1 & 1,334 & 1,213 & 0,121 \\
\hline c.5 & 2 & 1,085 & 0,92 & 0,165 \\
\hline c.5 & 3 & 1,56 & 1,604 & $-0,044$ \\
\hline c. 6 & 1 & 1,186 & 1,084 & 0,102 \\
\hline c. 6 & 2 & 1,16 & 1,136 & 0,024 \\
\hline c.6 & 3 & 1,459 & 1,332 & 0,127 \\
\hline c. 7 & 1 & 1,18 & 1,218 & $-0,038$ \\
\hline c.7 & 2 & 1,253 & 1,265 & $-0,012$ \\
\hline c.7 & 3 & 1,57 & 1,369 & 0,201 \\
\hline c. 8 & 1.2 .3 & 1,47 & 1,408 & 0,062 \\
\hline
\end{tabular}


Analizando los datos reflejados en la Tabla No8, la interpretación de Gastinel en esta parte A muestra otros patrones diferentes a lo expuesto sobre la versión de Casals. De los 25 pulsos, en 13 de ellos hay una duración mayor en la repetición frente a 12 pulsos de la primera interpretación. Se trata pues de un balance prácticamente similar en favor de una u otra entre ambas partes. Viendo el total de notas donde la duración limitada al decisegundo es de 0,0 , hay 17 contando con la blanca con puntillo separada en 3 partes. Por tanto, otra visión podría determinar que ese equilibrio entre las dos partes no es tal, y que hay una intencionalidad en conjunto de no diferenciar la primera interpretación de la repetición.
Respecto a los pulsos destacadamente diferenciados, se encuentra el tercer pulso del compás 5, así como el primer pulso del compás 6 . Ambos están seguidos, pero Gastinel no los destaca en el mismo lugar de su grabación, ya que el primero es largo en la repetición mientras que el segundo es largo en la primera interpretación. Así pues, ese alargamiento se desplaza un pulso hacia delante en la repetición frente a la primera interpretación. También se toma más tiempo en el primer pulso del compás 3 en la repetición.

Tabla 8

Duraciones de los pulsos en Gastinel. Parte A.

\begin{tabular}{ccccc} 
Compases & Pulso & Duración primera int. & Duración repetición & Diferencia \\
\hline c. 1 & 1 & 1,504 & 1,531 & $-0,027$ \\
\hline c. 1 & 2 & 1,102 & 1,074 & 0,028 \\
\hline c. 1 & 3 & 1,672 & 1,547 & 0,125 \\
\hline c. 2 & 1 & 1,381 & 1,449 & $-0,068$ \\
\hline c. 2 & 2 & 1,099 & 1,114 & $-0,015$ \\
\hline c. 2 & 3 & 1,499 & 1,402 & 0,097 \\
\hline c.3 & 1 & 1,295 & 1,548 & $-0,253$ \\
\hline c.3 & 2 & 0,948 & 0,867 & 0,081 \\
\hline c. 3 & 3 & 1,221 & 1,262 & $-0,041$ \\
\hline c. 4 & 1 & 1,243 & 1,247 & $-0,004$ \\
\hline c. 4 & 2 & 1,198 & 1,254 & $-0,056$ \\
\hline c. 4 & 3 & 2,017 & 2,029 & $-0,012$ \\
\hline c. 5 & 1 & 1,648 & 1,638 & 0,01 \\
\hline c. 5 & 2 & 0,903 & 1,005 & $-0,102$ \\
\hline c. 5 & 3 & 1,064 & 1,419 & $-0,355$ \\
\hline c. 6 & 1 & 1,369 & 1,102 & 0,267 \\
\hline c.6 & 2 & 0,946 & 1,013 & $-0,067$ \\
\hline c.6 & 3 & 1,187 & 1,166 & 0,021 \\
\hline c. 7 & 1 & 1,181 & 1,347 & $-0,166$ \\
\hline c. 7 & 2 & 1,162 & 1,125 & 0,037 \\
\hline c. 7 & 3 & 1,293 & 1,436 & $-0,143$ \\
\hline c. 8 & 1.2 .3 & 1,432 & 1,37 & 0,062 \\
\hline & & & & \\
\hline
\end{tabular}


Comparando ambas grabaciones y como se refleja en la Tabla No9, las diferencias se observan en cada pulso, destacando más la variedad que la falta de ella. En definitiva, hay más divergencias que convergencias. En la primera interpretación, las mayores discrepancias interpretativas (entiéndase), se dan entre la primera nota que Casals interpreta con una duración excepcional. Por otra parte, Gastinel opta por alargar el primer pulso del compás 5 con una duración de 0,314, mientras que Casals opta por hacerlo en el tercero con 0,496. En la repetición, Gastinel alarga el primer pul- so del tercer compás como novedad respecto a la primera interpretación, y Casals lo hace en el segundo. El tiempo que se toma Gastinel en el primer pulso del compás 5 (al igual que la primera interpretación), esta vez no se lo toma Casals en el tercero. El dato anecdótico se da en la diferencia en la nota final, porque a pesar que tanto Casals como Gastinel tienen diferentes duraciones para esta media de la blanca con puntillo tanto en la primera interpretación como en la repetición, la diferencia entre ambos es siempre la misma.

\section{Tabla 9}

Diferencias entre ambas interpretaciones respecto a la duración de los pulsos.

\begin{tabular}{|c|c|c|c|}
\hline Compases & Pulso & Duración primera int. & Duración repetición \\
\hline c. 1 & 1 & 0,428 & $-0,011$ \\
\hline c. 1 & 2 & 0,226 & 0,26 \\
\hline c. 1 & 3 & 0,136 & 0,11 \\
\hline c. 2 & 1 & 0,119 & $-0,019$ \\
\hline c. 2 & 2 & 0,258 & 0,142 \\
\hline c. 2 & 3 & 0,262 & 0,208 \\
\hline c.3 & 1 & 0,002 & $-0,304$ \\
\hline c.3 & 2 & 0,198 & 0,293 \\
\hline c.3 & 3 & 0,133 & 0,092 \\
\hline c. 4 & 1 & $-0,008$ & $-0,017$ \\
\hline c. 4 & 2 & 0,095 & $-0,01$ \\
\hline c. 4 & 3 & $-0,195$ & $-0,268$ \\
\hline c. 5 & 1 & $-0,314$ & $-0,425$ \\
\hline c. 5 & 2 & 0,182 & $-0,085$ \\
\hline c. 5 & 3 & 0,496 & 0,185 \\
\hline c. 6 & 1 & $-0,183$ & $-0,018$ \\
\hline c. 6 & 2 & 0,214 & 0,123 \\
\hline c. 6 & 3 & 0,272 & 0,166 \\
\hline c. 7 & 1 & $-0,001$ & $-0,129$ \\
\hline c. 7 & 2 & 0,091 & 0,14 \\
\hline c. 7 & 3 & 0,277 & $-0,067$ \\
\hline c. 8 & 1.2 .3 & 0,038 & 0,038 \\
\hline
\end{tabular}


Respecto a la parte $B$ y como se muestra en la Tabla $\mathrm{N}^{0} 10$, hay un total de 36 pulsos. En la versión que Pau Casals registró, estos pulsos marcan una diferencia acotada al centisegundo de 0,0 en 28 ocasiones, por lo que la constante vuelve a ser la igualdad dentro de que cada una es diferente. La excepción está en el segundo pulso del compás 13, donde, al milisegundo, ambos pulsos son iguales en la primera interpretación y en la repetición. Las diferencias más notables se dan en el primer pulso del compás 13 en la repetición, así como en el primer pulso del compás 9 que da

Tabla 10

Duraciones de los pulsos en Casals. Parte B.

\begin{tabular}{|c|c|c|c|c|}
\hline Compases & Pulso & Duración primera int. & Duración repetición & Diferencia \\
\hline c. 9 & 1 & 1,634 & 1,421 & 0,213 \\
\hline c. 9 & 2 & 1,256 & 1,166 & 0,09 \\
\hline c. 9 & 3 & 1,508 & 1,433 & 0,075 \\
\hline c. 10 & 1 & 1,354 & 1,222 & 0,132 \\
\hline c. 10 & 2 & 1,047 & 1,132 & $-0,085$ \\
\hline c. 10 & 3 & 1,514 & 1,459 & 0,055 \\
\hline c. 11 & 1 & 1,226 & 1,3 & $-0,074$ \\
\hline c. 11 & 2 & 1,047 & 1,119 & $-0,072$ \\
\hline c. 11 & 3 & 1,526 & 1,433 & 0,093 \\
\hline c. 12 & 1 & 1,258 & 1,255 & 0,003 \\
\hline c. 12 & 2 & 1,279 & 1,224 & 0,055 \\
\hline c. 12 & 3 & 1,523 & 1,404 & 0,119 \\
\hline c. 13 & 1 & 1,43 & 1,749 & $-0,319$ \\
\hline c. 13 & 2 & 1,096 & 1,096 & 0 \\
\hline c. 13 & 3 & 1,566 & 1,537 & 0,029 \\
\hline c. 14 & 1 & 1,238 & 1,206 & 0,032 \\
\hline c. 14 & 2 & 1,018 & 1,076 & $-0,058$ \\
\hline c. 14 & 3 & 1,343 & 1,381 & $-0,038$ \\
\hline c. 15 & 1 & 1,229 & 1,206 & 0,023 \\
\hline c. 15 & 2 & 1,169 & 1,226 & $-0,057$ \\
\hline c. 15 & 3 & 1,358 & 1,343 & 0,015 \\
\hline c. 16 & 1 & 1,203 & 1,197 & 0,006 \\
\hline c. 16 & 2 & 1,302 & 1,233 & 0,069 \\
\hline c. 16 & 3 & 1,575 & 1,546 & 0,029 \\
\hline c. 17 & 1 & 1,471 & 1,528 & $-0,057$ \\
\hline c. 17 & 2 & 1,192 & 1,224 & $-0,032$ \\
\hline c. 17 & 3 & 1,45 & 1,513 & $-0,063$ \\
\hline
\end{tabular}




\begin{tabular}{ccccc}
\hline c. 18 & 1 & 1,302 & 1,239 & 0,063 \\
\hline c. 18 & 2 & 1,204 & 1,279 & $-0,075$ \\
\hline c. 18 & 3 & 1,424 & 1,43 & $-0,006$ \\
\hline c. 19 & 1 & 1,2 & 1,25 & $-0,05$ \\
\hline c. 19 & 2 & 1,244 & 1,395 & $-0,151$ \\
\hline c. 19 & 3 & 1,412 & 1,577 & $-0,165$ \\
\hline c. 20 & 1 & 1,384 & 1,369 & 0,015 \\
\hline c. 20 & 2 & 1,449 & 1,766 & $-0,317$ \\
\hline c. 20 & 3 & 1,676 & 2,005 & $-0,329$ \\
\hline
\end{tabular}

Los datos de la parte B de la versión de la Sarabande que registró Anne Gastinel se pueden ver en la Tabla $N^{0} 11$. Aquí se observan cuatro pulsos a destacar notablemente. El primero de ellos es claramente el último pulso, donde se aprecia una diferencia a favor de la repetición de 0,954, cercana al segundo (lo que en una tercera lupa micro-agógica es una diferencia muy remarcable). Con una duración superior al medio segundo, con 0,672 , el primer pulso del compás 10 tiene una duración mayor en la primera interpretación: si Casals destacaba el primer pulso del compás 9, Gastinel lo hace en el 10. Como tercer punto destacable, el compás 12: el primer pulso destaca como largo en la primera interpretación $(0,323)$, el segundo en la repetición $(-0,223)$, y el tercer pulso es prácticamente similar $(0,028)$.

\section{Tabla 11}

Duraciones de los pulsos en Gastinel. Parte B.

\begin{tabular}{ccccc} 
Compases & Pulso & Duración primera int. & Duración repetición & Diferencia \\
\hline c. 9 & 1 & 1,42 & 1,479 & $-0,059$ \\
\hline c.9 & 2 & 1,144 & 1,279 & $-0,135$ \\
\hline c.9 & 3 & 1,523 & 1,501 & 0,022 \\
\hline c. 10 & 1 & 1,223 & 0,551 & 0,672 \\
\hline c. 10 & 2 & 0,986 & 1,114 & $-0,128$ \\
\hline c. 10 & 3 & 1,312 & 1,485 & $-0,173$ \\
\hline c. 11 & 1 & 1,266 & 1,46 & $-0,194$ \\
\hline c. 11 & 2 & 0,897 & 0,875 & 0,022 \\
\hline c. 11 & 3 & 1,227 & 1,474 & $-0,247$ \\
\hline c. 12 & 1 & 1,341 & 1,018 & 0,323 \\
\hline c.12 & 2 & 1,097 & 1,32 & $-0,223$ \\
\hline c.12 & 3 & 1,724 & 1,696 & 0,028 \\
\hline c.13 & 1 & 1,681 & 1,77 & $-0,089$ \\
\hline c. 13 & 2 & 0,985 & 0,967 & 0,018 \\
\hline c. 13 & 3 & 1,435 & 1,521 & $-0,086$ \\
\hline
\end{tabular}




\begin{tabular}{|c|c|c|c|c|}
\hline c. 14 & 1 & 1,45 & 1,531 & $-0,081$ \\
\hline c. 14 & 2 & 0,988 & 0,994 & $-0,006$ \\
\hline c. 14 & 3 & 1,249 & 1,36 & $-0,111$ \\
\hline c. 15 & 1 & 1,599 & 1,657 & $-0,058$ \\
\hline c. 15 & 2 & 0,973 & 0,949 & 0,024 \\
\hline c. 15 & 3 & 1,198 & 1,094 & 0,104 \\
\hline c. 16 & 1 & 1,502 & 1,335 & 0,167 \\
\hline c. 16 & 2 & 1,416 & 1,368 & 0,048 \\
\hline c. 16 & 3 & 0,97 & 1,144 & $-0,174$ \\
\hline c. 17 & 1 & 1,494 & 1,413 & 0,081 \\
\hline c. 17 & 2 & 1,077 & 1,023 & 0,054 \\
\hline c. 17 & 3 & 1,743 & 2,048 & $-0,305$ \\
\hline c. 18 & 1 & 1,433 & 1,35 & 0,083 \\
\hline c. 18 & 2 & 0,972 & 1,137 & $-0,165$ \\
\hline c. 18 & 3 & 1,3 & 1,342 & $-0,042$ \\
\hline c. 19 & 1 & 1,082 & 1,197 & $-0,115$ \\
\hline c. 19 & 2 & 1,066 & 1,297 & $-0,231$ \\
\hline c. 19 & 3 & 1,541 & 2,087 & $-0,546$ \\
\hline c. 20 & 1 & 1,348 & 1,51 & $-0,162$ \\
\hline c. 20 & 2 & 1,259 & 1,13 & 0,129 \\
\hline c. 20 & 3 & 1,864 & 2,818 & $-0,954$ \\
\hline
\end{tabular}

Para acabar, se compara finalmente esta parte B con los datos presentados en la Tabla No12. De los treinta y seis pulsos, ocho duran en la primera interpretación 0,0 al corte del centisegundo, y trece lo hacen en la repetición. Según estos datos, prima la variedad. Dentro de esa variedad, en la primera interpretación es de destacar que las diferencias no superan el 0,3 más que en dos ocasiones: la primera, de manera muy notable en el tercer pulso del compás 16, con 0,605 más tiempo en la interpretación de Pau Casals. La segunda, en el primer pulso del compás 15, con 0,37 más en la versión de Gastinel. En la repetición, con mucha más variedad y picos de diferencias, destaca con 0,607 la duración que Pau Casals le da por encima de la de Anne Gastinel al primer pulso del compás 10. Casals también se toma más tiempo en el tercer pulso del compás 16 (ya lo hizo en la primera interpre- tación), y en el penúltimo pulso, preparando el final. En cambio, Gastinel se toma su tiempo de más en el primer pulso del compás 14 con 0,325 (ya se tomó 0,212 en la primera parte), en el primer pulso del compás 15 con 0,451 (lo hizo en la primera parte con 0,37 ) y en el tercer pulso del compás 17 con 0,535 (lo hizo con menor intensidad en la primera interpretación con 0,293). Como novedad respecto a la primera interpretación, cambia el tercer pulso del compás 19, con una duración 0,51 mayor que la de Casals, que fue 0,129 en la primera interpretación. Finalmente, el pulso que destaca por encima de todos es el último, que coincide con la nota final, donde Gastinel se toma mucho más tiempo que Casals, concretamente 0,813 más. 
Tabla 12

Diferencias entre ambas interpretaciones respecto a la duración de los pulsos.

\begin{tabular}{|c|c|c|c|}
\hline Compases & Pulso & Duración primera int. & Duración repetición \\
\hline c. 9 & 1 & 0,214 & $-0,058$ \\
\hline c. 9 & 2 & 0,112 & $-0,113$ \\
\hline c. 9 & 3 & $-0,015$ & $-0,068$ \\
\hline c. 10 & 1 & 0,131 & 0,671 \\
\hline c. 10 & 2 & 0,061 & 0,018 \\
\hline c. 10 & 3 & 0,202 & $-0,026$ \\
\hline c. 11 & 1 & $-0,04$ & $-0,16$ \\
\hline c. 11 & 2 & 0,15 & 0,244 \\
\hline c. 11 & 3 & 0,299 & $-0,041$ \\
\hline c. 12 & 1 & $-0,083$ & 0,237 \\
\hline c. 12 & 2 & 0,182 & $-0,096$ \\
\hline c. 12 & 3 & $-0,201$ & $-0,292$ \\
\hline c. 13 & 1 & $-0,251$ & $-0,021$ \\
\hline c. 13 & 2 & 0,111 & 0,129 \\
\hline c. 13 & 3 & 0,131 & 0,016 \\
\hline c. 14 & 1 & $-0,212$ & $-0,325$ \\
\hline c. 14 & 2 & 0,03 & 0,082 \\
\hline c. 14 & 3 & 0,094 & 0,021 \\
\hline c. 15 & 1 & $-0,37$ & $-0,451$ \\
\hline c. 15 & 2 & 0,196 & 0,277 \\
\hline c. 15 & 3 & 0,16 & 0,249 \\
\hline c. 16 & 1 & $-0,299$ & $-0,138$ \\
\hline c. 16 & 2 & $-0,114$ & $-0,135$ \\
\hline c. 16 & 3 & 0,605 & 0,402 \\
\hline c. 17 & 1 & $-0,023$ & 0,115 \\
\hline c. 17 & 2 & 0,115 & 0,201 \\
\hline c. 17 & 3 & $-0,293$ & $-0,535$ \\
\hline c. 18 & 1 & $-0,131$ & $-0,111$ \\
\hline c. 18 & 2 & 0,232 & 0,142 \\
\hline c. 18 & 3 & 0,124 & 0,088 \\
\hline c. 19 & 1 & 0,118 & 0,053 \\
\hline c. 19 & 2 & 0,178 & 0,098 \\
\hline c. 19 & 3 & $-0,129$ & $-0,51$ \\
\hline c. 20 & 1 & 0,036 & $-0,141$ \\
\hline c. 20 & 2 & 0,19 & 0,636 \\
\hline c. 20 & 3 & $-0,188$ & $-0,813$ \\
\hline
\end{tabular}




\section{CONCLUSIONES}

Tal y como menciona David Blum en su trabajo sobre Pau Casals, parece ser que el intérprete catalán realizaba un exhaustivo trabajo nota por nota y a velocidad lenta que se refleja en la investigación presentada, por lo que el resultado final no es fruto de la improvisación. Casals como docente, transmitía la importancia de realizar este análisis micro-agógico, qué hacer con cada nota, una lupa que "no agrandaba el espacio sino el tiempo" (Blum $2000 a$, p. 111 ). Analizaba fragmentos de las Suites para decidir qué hacer en cada momento y fomentaba esta práctica de estudio a sus alumnos. Luego, una interpretación a tiempo real refleja sin duda el trabajo hecho a pequeña escala. Por los datos que se han extraído de la versión de Anne Gastinel, se puede afirmar que la violonchelista francesa realiza también una interpretación consciente y no fruto de la arbitrariedad o la improvisación.

En lo relativo a los datos obtenidos y a pesar de lo costoso de la medición de cada nota de manera manual con el programa Sonic Visualiser, la interpretación de los resultados muestra lo que se puede descubrir si el foco se pone en el detalle y no en el todo. Si bien la Sarabande no es un movimiento que se toque especialmente rápido, las diferencias agógicas más sutiles son difíciles de percibir a tiempo real. Tan solo un análisis de la micro-agógica y de las duraciones nota por nota puede arrojar luz sobre las pequeñas diferencias que se han presentado e interpretado, y que a priori, con una duración total similar, podrían tender a interpretarse como grabaciones con más convergencias que divergencias.

Analizando los aspectos que se han tratado, se puede ver que, si bien existen ciertas convergencias, ambas interpretaciones muestran dos maneras diferentes de tocar la Sarabande en un similar espacio de tiempo, dos interpretaciones diferentes en tres lupas de análisis con focos diferenciados. Hay más divergencias que convergencias, por pequeñas que estas sean. Incluso cabe afirmar que son cuatro maneras, si se incluyen las repeticiones. Con un silencio inicial y final prácticamente idéntico en ambas grabaciones y en una obra con un claro predominio de igualdad en los valores rítmicos, la riqueza que aporta el intérprete hace que las posibilidades sean grandes, lo que enfatiza su labor. En definitiva dos intérpretes, dos posibilidades diferentes, pero no opuestas. En el análisis de las partes $A-A^{\prime}$ y $B-B^{\prime}$, los datos muestran dos propuestas totalmente diferentes. A medida que la lupa se va acercando, las pequeñas convergencias y divergencias se multiplican. En la primera lupa, la de los grupos de 4 compases, hay una mayor igualdad general en Casals y una interpretación más irregular o variada en Gastinel. Son dos interpretaciones divergentes, pero la de Gastinel es más amplia por los extremos; de ella son el grupo más largo y el más corto. En esta primera lupa, se han podido observar algunas similitudes llamativas, como la escasa diferencia en el cuarto grupo de la primera interpretación y todavía más escasa diferencia en el segundo grupo de la repetición entre ambas interpretaciones. Respecto a la segunda lupa y en la versión de Pau Casals, en la parte $A$ hay una clara tendencia a una mayor duración en la primera interpretación respecto a la repetición, mientras que en $B$, la tendencia es mayoritariamente la contraria. Este claro patrón no se repite en la versión de Anne Gastinel, que no presenta ningún patrón claro en una versión más polarizada también en los parámetros de la segunda lupa. Ambas versiones de la Sarabande comparten la ubicación del compás de mayor duración, que es el último de la repetición que pone fin a la obra, por el tiempo que se toman en el ritardando final. No obstante, ese excepcional tiempo que se toman no es igual en ambas versiones, ya que en Gastinel es algo más de 1 segundo que en Casals, lo que en micro-agógica, es un tiempo sobresaliente.

Como menciona David Blum (2000a, p.80), "para Casals, la primera nota es la puerta por la que el impulso divino entra en nuestra esfera terrenal". Así se ve en la tercera lupa, donde Casals se toma un tiempo excepcionalmente largo en el primer pulso que da inicio a la obra. Es sabido que Casals le daba una especial importancia a la primera nota de la obra, y se ve en su duración: en sus propias palabras, "la primera nota siempre debe oír$\mathrm{se}^{\prime \prime}$ (Blum 2000b, p. 168). Casals defendía que esta primera nota debía ser más larga que el resto, optando por destacarla por su duración: "esto da una carácter de rubato muy agradable, lo que no quiere decir que sea rubato. No debemos oír una nota tras otra como si fuera una máquina" (Blum 2000a, p. 150). Por lo demás, y dadas las escasas diferencias en los datos obtenidos, no parece que haya una especial intención por parte de Casals en alargar los pulsos en la primera interpretación o en la repetición. En cambio, Gastinel reparte 13 a 
12 la mayor duración en la repetición y en la primera interpretación respectivamente, si bien los escasos márgenes de este equilibrio bien pudieran dar una lectura similar a la realizada en la interpretación de Casals.

Las dos interpretaciones de la Sarabande, compartiendo una similar duración, están llenas de sutiles convergencias y divergencias en una obra donde, como se ha destacado, de 108 notas, 100 tienen el valor rítmico de corchea, lo que en un primer término presenta un punto de partida poco variado. Nada más lejos de la realidad: variedad frente a repetición, divergencias frente a convergencias, diferencias frente a similitudes. Respetando la partitura de Bach, se visualiza el amplio margen que hay para la interpretación y del que tan buen uso hacen tanto Anne Gastiel como Pau Casals.

\section{BIBLIOGRAFÍA}

Alavedra, J. (1975). Pau Casals. Barcelona: Editorial Aedos.

Baldock, R. (1994). Pau Casals. Barcelona: Paidós testimonios.

Blum, D. (2000a). Casals y el Arte de la Interpretación. Barcelona: Idea Books.

(2000b). El arte del cuarteto de cuerda. El Cuarteto Guarneri en conversación con David Blum. Barcelona: Idea Books.

Corredor, J. M. (1975). Pablo Casals nos cuenta su vida. Conversaciones con el maestro. Barcelona: Editorial Juventud.

Eisenberg, M. (1943). Casals and the Bach Suites. Violins and Violinists, 1-2.

Gardner, C. (3 de junio de 2008). Too young to play Bach? There is no need for an age limit on playing the cello suites. The Guardian. Recuperado de https://www.theguardian.com/music/musicblog/2008/jun/03/ tooyoungtoplaybach

Heppstein, H. (ed.) (1988). Sechs Suiten für Violoncello solo. Kassel: Bärenreiter Verlag. Recuperado de http:// imslp.eu/files/imglnks/euimg/f/f6/ IMSLP480717-PMLP164353-bachNBAVI,2suiteV(c-Moll)BWV1011 textl.pdf
Kemp, L. (junio de 2008). Bach Cello Suites. Passionate about Bach but maybe this loving player is a touch too intense. Gramophone. Recuperado de https://www.gramophone. co.uk/review/bach-cello-suites-3

Saenz, I. (2017a). Pau Casals (1876-1973), el virtuoso autodidacta. Artseduca, 16, 110 129.

(2017b). Pau Casals y el re-descubrimiento de las Suites para violoncello solo de J.S. Bach. El Artista, 14, 83-93.

(2018a). Casals y Bach: análisis performativo de la agógica en la Sarabande BWV 1011. Quodlibet, Revista de Especialización Musical, 67, 50-65.

(2018b). Herramientas "software" para la interpretación musical. Un método de análisis para descubrir la micro-agógica. Journal of Sound, Silence, Image and Technology, 1, 14-23.

Siblin, E. (2011). Las Suites para violonchelo. En busca de Pau Casals, J.S. Bach y una obra maestra. Madrid: Turner Música.

Talbot, J. (31 de mayo de 2008). Bach: Solo Cello Suites BWV1007-12. The Strad. Recuperado de https://www.thestrad.com/ bach-solo-cello-suites-bwv1 007-12/621. article

Zurita, T. (2015). La interpretación del violonchelo romántico: de Paganini a Casals. Barcelona: Antoni Bosch editor. 